[Type text]

\title{
Development and validation of a VISA tendinopathy questionnaire for Greater Trochanteric Pain
} Syndrome, the VISA-G

Fearon AM ${ }^{1,2,}$ Ganderton $C^{3}$, Scarvell JM ${ }^{2,5}$, Smith PN ${ }^{1,2,}$, Nash C ${ }^{4}$, Cook JL ${ }^{4}$

${ }^{1}$ ANU Medical School, College of Medicine, Biology and Environment, Australian National University, Canberra, Australia; ${ }^{2}$ Trauma and Orthopaedic Research Unit, the Canberra Hospital, Canberra, Australia; ${ }^{3}$ School of Physiotherapy, Faculty of Health Science, La Trobe University, Melbourne, Australia; ${ }^{4}$ Department of Physiotherapy, School of Primary Health Care, Monash University, Melbourne, Australia; ${ }^{5}$ Discipline of Physiotherapy, Faculty of Health, University of Canberra, Canberra, Australia.

Corresponding author:

Angela Fearon

Trauma and Orthopaedic Research Unit

The Canberra Hospital

PO Box 11, Woden, ACT

Australia, 2606.

Email: Afearon.au@Gmail.com

Phone: +61 468494799

Key words: Tendinopathy, patient outcome assessment, GTPS, Gluteus muscles.

Word count $=2627$ 
[Type text]

\section{ABSTRACT}

Background Greater trochanteric pain syndrome (GTPS) is common, resulting in significant pain and disability. There is no condition specific outcome score to evaluate the degree of severity of GTPS in or effectiveness of treatment for, patients with this condition.

Objective To develop a reliable and valid outcome tool to measurement the severity of GTPS, so as to be able to evaluate treatments.

Methods An iterative process of item generation, clarification, reduction was used to develop this outcome tool, the VISA-G. The VISA-G was tested for reliability (ICC), internal consistency (Cronbach's Alpha), and construct validity (correlation co-efficient) on 52 naïve participants with GTPS and 31 asymptomatic participants.

Results The resultant outcome tool is consistent in style with existing tendinopathy outcome tools, namely the suit of VISA scores. The VISA-G was found to be have a test retest reliability of ICC $_{2,1}$ ( $95 \%$ $\mathrm{Cl}$ ) of 0.827 (0.638 to 0.779 ). Internal consistency was high with a Cronbach's Alpha of 0.809 . Construct validity was demonstrated: the VISA-G measures different constructs than both the HHS and the ODI (Spearman Rho:0.020 and0.0205 respectively). The VISA-G did not demonstrate any floor or ceiling effect in symptomatic participants.

Conclusion The VISA-G has is a reliable and valid score for measuring the severity of GTPS. 
[Type text]

\section{INTRODUCTION}

Greater trochanteric pain syndrome (GTPS) is a common, chronic, disabling condition characterised by intermittent or continuous pain at, or around, the greater trochanter of the femur.[ll 23 3 The condition can be debilitating enough to reduce activity and capacity for employment.[4] GTPS has a reported incidence of 1.8 patients per 1000 per year in primary care.[5] Prevalence rates of $23.5 \%$ in women at risk of knee osteoarthritis [6] and 54\% in recipients of renal transplants [7] have been reported. These statistics likely underestimate the prevalence of GTPS as misdiagnosis of GTPS is common.[8-11]

Risk factors for developing GTPS are poorly understood however female gender, older age,[1 6] greater adiposity, [6] back pain [4 6] and a higher femoral neck angle [12] have been associated with the condition. Older women are over represented in GTPS by a ratio of approximately $4: 1,[56]$ with the reported age of diagnosis of between 45 and 63 years. [1 5 6]

Despite the reported prevalence and disability, a condition specific patient reported outcome (PRO) tool has not been developed for this condition. Surrogate measures using tools designed for hip osteoarthritis or low back pain have been used.[1 4 13] Evaluation of the level of severity, and of the effectiveness of treatment across studies would be enhanced by the use of a PRO.[14 15]

The VISA questionnaires have a consistent structure has been shown to be valid for the assessment of lower limb tendinopathies. The VISA-P, VISA-A and VISA-H have been widely adopted to quantify the disability associated with patellar, Achilles and hamstring tendinopathy respectively.[16-18] Additionally, their purpose is to monitor and evaluate treatment strategies for these conditions.[19] As GTPS is a tendinopathy involving the gluteus medius and minimus tendons, [20-24] the VISA questionnaire structure may be a valid to adopt for this population. The objective of this study was to develop a valid PRO questionnaire, based on the VISA questionnaire structure, for people with GTPS. The development of the VISA-G followed the Consensus-based Standards for the selection of 
[Type text]

health Measurement Instruments (COSMIN) guidelines for the development and testing of health measurement instruments provide a methodology for the development health measurement tools.[25]

\section{METHODS}

The development of this instrument used qualitative and quantitative methods using three steps:

(1) item generation and format refinement, (2) item reduction, and (3) evaluation of the psychometric properties of the final version.[26] Existing VISA questionnaires were used to guide the format of the VISA-G.[16-18]

\section{Participants}

Participants were recruited via social and professional networks. Participants were screened for exclusion criteria and then provided with a comprehensive participant information sheet before providing written consent. Ethical approval for this study was provided by ACT health human research ethics committee, Australian National University human research ethics committee, Calvary health care ACT human research ethics committee, Deakin University human research ethics committee, and the Monash University human research ethics committee.

Inclusion criteria

GTPS participants:

In the item generation stage of this research GTPS participants were identified by clinician as having trochanteric bursitis and / or gluteal tendinopathy as the primary diagnosis. In subsequent stages GTPS was defined as a history of lateral hip pain,[2 362024 27-30] pain on palpation of the greater trochanter,[1 223202829 31] and one or both of lateral hip pain with lying on the ipsilateral side,[1 2931 ] or during weight bearing activities or sitting.[2 1131 ]

Asymptomatic participants 
[Type text]

Participants were included in the asymptomatic group if they were healthy with no current or history of back or hip pain requiring them to seek treatment for the condition.

\section{Exclusion criteria}

Participants were excluded if they had any signs or symptoms of hip OA based on Altman criteria [33], systemic inflammatory disease e.g. rheumatoid arthritis; lumbar spine nerve root signs; a history of lumbar spine or ipsilateral hip joint surgery; osteogenic disease e.g. Paget's disease; or a corticosteroid injection to the ipsilateral lateral hip region in the preceding three months. Participants with co-morbidities that restricted their activity level more than their hip pain did - thus invalidating question eight (Q8), were also excluded.

Health professional inclusion

Physiotherapists were invited to attend two focus groups. Fifteen to 20 physiotherapists were present at each meeting with experience ranging from new graduate to extensive (post graduate musculoskeletal training and more 20 years of experience). No physiotherapist was excluded.

One sports physician, two orthopaedic surgeons and a physician with a post graduate diploma in ultrasound imaging, all with an interest in the condition were involved in the item generation on a one-to-one discussion basis.

\section{Process}

Step 1 Item generation

Item generation was undertaken using a number of methods: A literature review identified items noted in the literature; focus groups of physiotherapists discussed the literature review findings in addition to their personal observations of presentation and dysfunction of clients with GTPS; and semi-structured interviews of people with GTPS $(\mathrm{N}=42)$. The information from the literature review, the focus groups and the in depth interviews provided triangulation of data.[32] These data were condensed into a draft questionnaire. Trial questionnaires were sent to interview participants for 
[Type text]

testing of question accuracy, clarity and questionnaire design.[33] This was done in an iterative fashion - until no new domains were elicited. The subsequent iteration was trialled on 73 naive GTPS participants. These data informed the item reduction.

Step 2 Item reduction

Item reduction was undertaken by two experienced research physiotherapists (AF \& JC). The results from the questionnaire were evaluated for domain generation using principal component analysis and scree plots, and for redundancy using Spearman Rho correlation. In this early version, ceiling and floor effects were tested via descriptive statistics. This process resulted in a reduction from 21 to seven questions. In line with the VISA questionnaire model two questions regarding level of activity (Q7 \& Q8) were added to account for variation in tendon pain due to variation in tendon loading. [17 18]

Step 3 Item scaling

As with existing VISA scores, the first six questions were scored between 0 and 10, while the categorisation questions ( 7 and 8 ) were scored out of 10 and 30 respectively.

Step 4 Evaluation of psychometric properties

The final version of the VISA-G had a visual analogue score for pain ( $0=$ worst pain, $10=$ no pain), four questions related to pain (Questions 2,3, 4 and 6), one question related to difficulty with moving after sitting (Question 5) and two activity related questions (Questions 7 and 8). This version of the VISA-G (Attachment A) was tested on 52 naïve participants with GTPS; 49 women (mean age (SD)) 58.9 (13.64), and 3 men (53.0, SD 15.13). In addition 31 asymptomatic participants were tested: 24 women (57.4 (5.59) and 7 men (58.4 (5.22). Three asymptomatic participants did not specify their gender or age on the questionnaire.

Internal consistency: Internal consistency is the degree to which the items in questionnaire are interrelated.[25] A principal component factor analysis was performed on the individual items to 
[Type text]

determine their structural validity. A Cronbach's alpha and 95\% confidence intervals was calculated for each item and a score above 0.70 was taken as a sufficient level of homogeneity among the items.[34]

Test retest reliability: An intraclass correlation coefficient (ICC) was used to compare VISA-G scores of 26 GTPS participants who completed the PROs one week apart.

Face, content and construct validity: The VISA-G was developed following wide consolation with symptomatic participants, and health professionals. Content validity was assessed via assessment against the Harris Hip Score (HHS) and the Oswestry Disablity Index (ODI) - two scores that have previously been used in GTPS research and no reference standard exists to test the VISA-G against.[1 4 35] Construct validity was calculated to determine if the VISA-G correlated with the Harris Hip Score (HHS) and the Oswestry Disability Index (ODI) scores using a Spearman's Rank correlation coefficient.[1 4] .

Sample size calculation: Using the pain measure as the measure to determine the sample size, assuming a minimally significant difference of 1.5 points on the VAS [36], a power of 90 , and an alpha of 0.05 , the expected sample size required is 38 . Using the larger pain difference of two points, and the same parameters, results in a sample size of 20 for each group.

Floor and Ceiling effects: Floor and ceiling effects were examined by evaluating the distribution of total scores and determining if more than $15 \%$ of the participants achieved either the lowest or the highest score.

\section{Data analysis}

Statistical analysis for data reduction was undertaken using Stata IC 10.1 (Statacorp, USA). Statistical analysis for stability and groups differences was undertaken using SPSS Statistics (version 19, IBM SPSS Inc. Chicago, IL, USA). 
[Type text]

Eighty three complete questionnaires were used in the analysis, no imputation was used for missing items.

Item reduction: Multivariate techniques including factor analysis, bi-plots and Spearman rho correlation coefficients were employed. Using Factor Analysis results, two researchers reviewed the eigenvalues and loading factor for each question in VISA-Gv1. Biplots were used to examine the variance of each question, a higher variance was assumed to be associated with lower specificity of the domain. Spearman rho correlation co-efficient in combination with the factor analysis was used to identify items that appeared to measure the same construct, thus identifying redundancy and allowing question deletion. For example the level of discomfort associated with pushing a shopping trolley will depend upon how full the trolley is, likewise, the discomfort associated with getting in or out of a car will depend on which leg is affected and whether you are the driver or the passenger. We retained the five questions that covered $95 \%$ of the variance in the questionnaire. Thus we reduced the questionnaire from 22 questions to six (the five plus the pain VAS). To account for variation in tendon pain due to variation in tendon loading,[17 18] questions regarding level of activity (similar to VISA-A and VISA-P) was added to the final version (questions 7 and 8).

\section{Results}

Participants with GTPS had scores associated with higher levels of pain and dysfunction than the asymptomatic participants (Table 1).

Table 1: Result from the GTPS and asymptomatic participants for the VISA-G, Harris Hip Score and the Oswestry Disablity Index.

\begin{tabular}{|c|c|c|c|}
\hline Instrument & $\begin{array}{c}\text { GTPS participants } \\
\text { (Mean, SD) }\end{array}$ & $\begin{array}{l}\text { Asymptomatic participants } \\
\text { (Mean, SD) }\end{array}$ & T-Test \\
\hline & $N=52$ & $N=31$ & \\
\hline
\end{tabular}


[Type text]

\begin{tabular}{llll}
\hline VISA-G & $47.00(13.35)$ & $99.84(0.64)$ & $<0.001$ \\
HHS & $66.50(13.48)$ & $89.32(4.38)$ & $<0.001$ \\
ODI & $11.33(7.85)$ & $0.26(0.77)$ & $<0.001$
\end{tabular}

Note: VISA-G = VISA score for GTPS; HHS = Harris Hip Score; ODI = Oswestry Disability Index High scores for VISA-G and HHS indicate less disability. Lower scores for the ODI indicate less disability.

\section{Psychometric Testing}

Test-retest reliability: The VISA-G demonstrated a high level of reliability: $\mathrm{ICC}_{2,1}(95 \% \mathrm{Cl})$ of 0.827 (0.638 to 0.779$)$.

Internal consistency: Initial analysis resulted in a Cronbach's Alpha (95\% Cl) of $0.52(0.29-0.71)$ for the eight items. Post hoc stepwise item elimination yielded a Cronbach's Alpha range of 0.377 to 0.809. Deletion of question 8 resulted in the biggest change to the Cronbach's Alpha; increasing it to 0.809. The principal components analysis revealed that questions one to seven contributed evenly to the outcome of the score, with a range of mean (SD) of between 6.43 (3.84) and 8.30 (2.48). Question eight had a mean (SD) of 14.64 (13.28).

Validity

Face validity: The extensive item generation, logical item reduction, and the rigorous trialling over time with naive populations of symptomatic and asymptomatic people confers face validity.

Construct validity: Spearman correlation coefficient analysis illustrates the correlations between the VISA-G, HHS and ODI (Table 2). In participants with GTPS, the VISA-G did not correlate with either the HHS or the ODI, while in the asymptomatic group the VISA-G did correlate with the HHS, but not the ODI.

Table 2. Construct Validity (Spearman Rho's correlation co-efficient).

$\begin{array}{llll}\text { Group } & \text { VISA-G } & \text { HHS } & \text { ODI }\end{array}$


[Type text]

\begin{tabular}{lcccc}
\hline VISA G & GTPS & 1.000 & 0.020 & 0.205 \\
& Asymptomatic & 1.000 & $0.410^{*}$ & -0.300 \\
& & & & \\
\hline HHS & GTPS & 0.020 & 1.000 & $-0.778^{\dagger}$ \\
& Asymptomatic & $0.410^{*}$ & 1.000 & -0.149 \\
& GTPS & 0.205 & $-0.778^{\dagger}$ & 1.000 \\
& Asymptomatic & -0.300 & -0.149 & 1.000 \\
& & & & \\
\hline
\end{tabular}

$* p=0.022 ;+p \leq 0.001$.

VISA-G = VISA score for GTPS; HHS = Harris Hip Score; ODI = Oswestry Disability Index

Content Validity: The development process for the VISA-G, including people with GTPS and two

forms of clinicians, suggests that the questionnaire has content validity.

Floor and ceiling effects: No floor or ceiling effects were identified in the GTPS group with less than $15 \%$ of participants in the GTPS groups scoring the minimum or maximum values. Not surprisingly, $94 \%$ of the asymptomatic group scored the maximum value.

\section{Discussion}

The objective of this study was to develop a reliable and valid PRO questionnaire to assess the severity of, and efficacy of treatment for, GTPS. We have demonstrated that the VISA-G has good reliability - both internally and on a test-retest basis. The VISA-G has been shown to have content and construct validity. Concurrent criterion validity has been demonstrated, although not predictive criterion validity, thus meeting the majority of the COSMIN standards. Further research is needed to complete the process of demonstrating responsiveness and minimal clinical significance.

The validity of the VISA-G was assessed with the rigorous and recursive nature of the initial item generation and thorough item identification. While some of the questions overlap with the Harris Hip Score,[37] the scores from HHS and the VISA-G did not correlate, suggesting that the two scores 
[Type text]

are measuring different constructs. The high internal consistency further supports this, as does the result that people with GTPS scored higher on the VISA-G score than the control group.

The VISA-G has internal consistency for the questions that evaluated the GTPS construct (Q $1-6)$. The remaining questions modulate the effect of activity on the total score, consistent with the standard VISA format.[17 18 38] The HHS, ODI and the non-arthritic hip score [39] do not have such influential questions.

The activity level question ( $Q$ 8) in existing VISA scores focus on sport and training activities. Our item generation did not identify sport as a major activity of people with GTPS. Rather, walking and shopping were more common activities impacted on by GTPS. We amended this question in the VISA-G to weight bearing activities. This move away from sport related activities reflects the different age group that is represented in population with GTPS, as opposed to the younger and more active population with patellar tendinopathy particularly,[18] Achilles tendinopathy [17] and hamstring tendinopathy.[38]

The VISA-G is a measure of severity of GTPS and is not diagnostic, diagnosis should be clinical with a history and examination, including using the FABER test.[40] Patients with GTPS are likely to have comorbidities such as pain generated from the back and hip joint and the VISA-G score will likely be influenced by these. Interestingly, GTPS participants with non-specific low back pain (without referral) were included in this study, yet the correlation with the ODI in this population was not significant. This suggests that the VISA-G is responsive to GTPS independently of back pain symptoms. This is noteworthy when considering the study that treated GTPS in people diagnosed with back pain, and found an improvement in the ODI score.[13]

The VISA-G is modelled on existing VISA tendon outcome tools.[17 18 38] The VISA-G follows the standard VISA format, importantly including measures of activity level (Questions 7 and 8). It is expected that in line with other VISA tools Q1 -6 are likely to be very responsive to change, but Q7 and 8 likely to be slower - thus re-testing should only be done on a monthly or bi-monthly period. 
[Type text]

\section{Limitations}

A limitation of this study was that the GTPS participants represent people seeking conservative physiotherapy treatment. GTPS can be treated with corticosteroid injections and tendon reconstructions, [41 42] thus the VISA-G has not been tested a cross the full spectrum of a population with GTPS. In addition, testing of responsiveness and establishing minimum clinical significance was not possible with a cross-sectional study design.

\section{Conclusion}

This study found that the VISA-G is reliable and valid. It will be a useful tool for evaluating the severity of disability in, and the effectiveness of treatment for people with GTPS.

\section{WHAT ARE THE NEW FINDINGS}

1. A Valid and reliable tool for measuring the severity of GTPS.

2. VISA-G provides a tool for measuring the effectiveness of treatment for GTPS in both clinical and research settings.

\section{HOW MIGHT THIS IMPACT OF CLINICAL PRACTICE IN THE NEAR FUTURE}

- The VISA-G provides clinicians with an object method of measuring changes in people with GTPS, thus informing ongoing treatment.

- The VISA-G provides researchers with an object method of measuring changes in people with GTPS, thus making the results of research more convincing.

\section{ACKNOWLEDGEMENTS}

The VISA - G was developed at the Trauma and Orthopaedic Research Unit at the Canberra Hospital, with reliability and validity testing undertaken at Monash University.

Physiotherapist from the Canberra region and Doctors Rob Reid, Alexander Burn and Wes Cormick helped with the item generation.

Dr T Neeman from the Statistical Consulting Unit, the Australian National University, assisted with the item reduction.

The study was funded through Australian National University research funding and Monash University grants..... 
1. Rompe J, Segal N, Cacchio A, et al. Home Training, Local Corticosteroid Injection, or Radial Shock Wave Therapy for Greater Trochanter Pain Syndrome. American Journal of Sports Medicine 2009;37(10):1981-90 doi: 10.1177/0363546509334374[published Online First: Epub Date]|.

2. Gordon EJ. Trochanteric bursitis and tendinitis. Clinical Orthopaedics 1961;20:193-202

3. Anderson TP. Trochanteric bursitis: diagnostic criteria and clinical significance. Archives of Physical Medicine and Rehabilitation 1958;39(10):617-22

4. Fearon A, Cook J, Scarvell J, et al. Greater trochanteric pain syndrome negatively affects work, physical activity and quality of life: a case control study. Journal of Arthroplasty 2014;29(2):383- 86

5. Lievense A, Bierma-Zeinstra S, Schouten B, et al. Prognosis of trochanteric pain in primary care. The British Journal of General Practice 2005;55(512):199-204

6. Segal NA, Felson DT, Torner JC, et al. Greater trochanteric pain syndrome: epidemiology and associated factors. Archives of Physical Medicine and Rehabilitation 2007;88(8):988-92 doi: 10.1016/j.apmr.2007.04.014[published Online First: Epub Date]|.

7. Demant AW, Kocovic L, Henschkowski J, et al. Hip Pain in Renal Transplant Recipients: Symptomatic Gluteus Minimus and Gluteus Medius Tendon Abnormality as an Alternative MRI Diagnosis to Avascular Necrosis. American journal of roentgenology 2007;188(2):515-19 doi: 10.2214/ajr.05.1097[published Online First: Epub Date]|.

8. Collee G, Dijkmans BA, Vandenbroucke JP, et al. A clinical epidemiological study in low back pain. Description of two clinical syndromes. British Journal of Rheumatology 1990;29(5):354-7

9. Cormier G, Berthelot JM, Maugars Y. Gluteus tendon rupture is underrecognized by French orthopedic surgeons: results of a mail survey. Joint Bone Spine 2006;73(4):411-3 doi: S1297319X(06)00136-9 [pii] 10.1016/j.jbspin.2006.01.021[published Online First: Epub Date]|.

10. Lequesne M. From "periarthritis" to hip "rotator cuff" tears. Trochanteric tendinobursitis. Joint Bone Spine 2006;73(4):344-8 doi: 10.1016/j.jbspin.2007.12.004[published Online First: Epub Date]|.

11. Tortolani P, Carbone J, Quartararo L. Greater trochanteric pain syndrome in patients referred to orthopedic spine specialists. The Spine Journal 2002;2(4):251-4

12. Fearon A, Stephens S, Cook J, et al. The relationship of femoral neck shaft angle and adiposity to greater trochanteric pain syndrome in women. A case control morphology and anthropometric study. Br J Sports Med 2012;46(12):888-92 doi: 10.1136/bjsports-2011090744[published Online First: Epub Date]|.

13. Sayegh F, Potoupnis M, Kapetanos G. Greater trochanter bursitis pain syndrome in females with chronic low back pain and sciatica. Acta Orthopaedica Belgica 2004;70(5):423-28

14. Kirkley A, Griffin S, McLintock H, et al. The development and evaluation of a disease-specific quality of life measurement tool for shoulder instability. The Western Ontario Shoulder Instability Index (WOSI). Am J Sports Med 1998;26(6):764-72

15. Thomee $P$, Wahrborg $P$, Borjesson $M$, et al. A new instrument for measuring self-efficacy in patients with an anterior cruciate ligament injury. Scand J Med Sci Sports 2006;16(3):181-7 doi: 10.1111/j.1600-0838.2005.00472.x[published Online First: Epub Date]|.

16. Cacchil A, De Paulis F, Maffulli N. Development and validation of a new visa questionnaire (VISAH) for patients with proximal hamstring tendinopathy. Br. J. Sports Med. 2014;48(6):448-52 doi: 10.1136/bjsports-2012-091552.[published Online First: Epub Date]|.

17. Robinson JM, Cook JL, Purdam C, et al. The VISA-A questionnaire: a valid and reliable index of the clinical severity of Achilles tendinopathy. Br. J. Sports Med. 2001;35(5):335-41

18. Visentini PJ, Khan KM, Cook JL, et al. The VISA score: an index of severity of symptoms in patients with jumper's knee (patellar tendinosis). Victorian Institute of Sport Tendon Study Group. J Sci Med Sport 1998;1(1):22-8 
[Type text]

19. Khan KM, Visentini PJ, Kiss ZS, et al. Correlation of ultrasound and magnetic resonance imaging with clinical outcome after patellar tenotomy: prospective and retrospective studies. Victorian Institute of Sport Tendon Study Group. Clin J Sport Med 1999;9(3):129-37

20. Lequesne $M$, Mathieu $P$, Vuillemin-Bodaghi V, et al. Gluteal tendinopathy in refractory greater trochanter pain syndrome: diagnostic value of two clinical tests. Arthritis Care and Research 2008;59(2):241-6 doi: 10.1002/art.23354[published Online First: Epub Date]|.

21. Fearon A, Twin J, Dahlstrom J, et al. Increased substance P expression in the trochanteric bursa of patients with greater trochanteric pain syndrome. Rheumatol Int 2014 doi: 10.1007/s00296-041-2957-7[published Online First: Epub Date]|.

22. Connell DA, Bass C, Sykes CA, et al. Sonographic evaluation of gluteus medius and minimus tendinopathy. European Radiology 2003;13(6):1339-47

23. Fearon AM, Scarvell JM, Cook JL, et al. Does ultrasound correlate with surgical or histologic findings in greater trochanteric pain syndrome? A pilot study. Clinical Orthopaedics and Related Research 2010;468(7):1838-44 doi: 10.1007/s11999-009-1174-2[published Online First: Epub Date]|.

24. Woodley SJ, Nicholson HD, Livingstone V, et al. Lateral hip pain: findings from magnetic resonance imaging and clinical examination. The Journal of Orthopaedic and Sports Physical Therapy 2008;38(6):313-28

25. Mokkink LB, Terwee CB, Patrick DL, et al. COSMIN Checklist for the evaluating the methodological quality of studies on measurement properties: A clarification of its content. . BMC Med Res Methodol 2010;10:22

26. Dizon JMR, Grimmer-Somers K, Kumar S. The physical therapy profile questionnaire (PTPQ): development, validation and pilot testing. BMC research notes 2011;4:362- doi: doi: 10.1186/1756-0500-4-362[published Online First: Epub Date]|.

27. Shbeeb MI, O'Duffy JD, Michet CJ, Jr., et al. Evaluation of glucocorticosteroid injection for the treatment of trochanteric bursitis. Journal of Rheumatology 1996;23(12):2104-6

28. Bird PA, Oakley SP, Shnier R, et al. Prospective evaluation of magnetic resonance imaging and physical examination findings in patients with greater trochanteric pain syndrome. Arthritis and Rheumatism 2001;44(9):2138-45

29. Ege-Rasmussen K, Fan $\varnothing$ N. Trochanteric Bursitis. Scandinavian Journal of Rheumatology 1985;14:417- 20

30. Karpinski MR, Piggott H. Greater trochanteric pain syndrome. A report of 15 cases. The Journal of Bone and Joint Surgery. British volume 1985;67(5):762-3

31. Schapira D, Nahir M, Scharf Y. Trochanteric bursitis: a common clinical problem. Archives of Physical Medicine and Rehabilitation 1986;67(11):815-7 doi: 0003-9993(86)90164-4 [pii][published Online First: Epub Date]|.

32. Guion L, Diehl D, McDonald D. Triangulation: Establishing the validity of qualitative studies. 2nd ed: University of Florida - IFAS extension, 2011.

33. Fairbank JC, Pynsent PB. The Oswestry Disability Index. Spine (Phila Pa 1976) 2000;25(22):294052

34. Bland JM, Altman DG. Statistics notes: Cronbach's alpha. BMJ 1997;314(7080):572 doi: 10.1136/bmj.314.7080.572[published Online First: Epub Date]|.

35. Furia JP, Rompe JD, Maffulli N. Low-energy extracorporeal shock wave therapy as a treatment for greater trochanteric pain syndrome. The American Journal of Sports Medicine 2009;37(9):1806-13 doi: 10.1177/0363546509333014[published Online First: Epub Date]|.

36. Kelly A-M. The minimum clinically significant difference in visual analogue pain score does not differ with severity of pain. Emergency medicine 2001;18:205 - 07

37. Harris WH. Traumatic arthritis of the hip after dislocation and acetabular fractures: treatment by mold arthroplasty. An end-result study using a new method of result evaluation. J Bone Joint Surg Am 1969;51(4):737-55 
[Type text]

38. Cachhio A, Fosco DP, Maffulli N. Development and validation of a new visa quesionnaire (VISA-H) for patients with proximal hamstring tendinopathy. Br. J. Sports Med. 2013 doi: 19.1136/bjsports-2012-091552[published Online First: Epub Date]|.

39. Christensen CP, Althausen PL, Mittleman MA, et al. The nonarthritic hip score: reliable and validated. Clin Orthop Relat Res 2003(406):75-83

40. Fearon AM, Scarvell JM, Neeman T, et al. Greater trochanteric pain syndrome: defining the clinical syndrome. Br J Sports Med 2013;47(10):649-53 doi: 10.1136/bjsports-2012091565[published Online First: Epub Date]|.

41. Brinks A, van Rijn RM, Willemsen SP, et al. Corticosteroid Injections for Greater Trochanteric Pain Syndrome: A Randomized Controlled Trial in Primary Care. Annals of Family Medicine 2011;9(3):226-34 doi: 10.1370/afm.1232[published Online First: Epub Date]|.

42. Walsh N, Walsh M, Walton J, et al. Surgical repair of the abductor mechanism of the hip. J Bone Joint Surg Br 2011;93(B: supp) 